OPEN ACCESS

Edited by:

Ying $X u$,

University at Buffalo,

United States

Reviewed by:

Yaping Yan,

Shaanxi Normal University, China

Guang-Xian Zhang,

Thomas Jefferson University,

United States

Zengqiang Yuan,

Institute of Basic Medical Sciences

(CAMS), China

*Correspondence:

Bao-Guo Xiao

bgxiao@shmu.edu.cn

Cun-Gen Ma

macungen2001@163.com

Received: 10 August 2019 Accepted: 12 December 2019

Published: 08 January 2020

Citation:

Yin J-J, He Y, An J, Miao Q, Sui R-X, Wang $Q$, Yu J-Z, Xiao B-G and Ma C-G (2020) Dynamic Balance of Microglia and Astrocytes Involved in the Remyelinating Effect of Ginkgolide B.

Front. Cell. Neurosci. 13:572. doi: 10.3389/fncel.2019.00572

\section{Dynamic Balance of Microglia and Astrocytes Involved in the Remyelinating Effect of Ginkgolide B}

\author{
Jun-Jun Yin ${ }^{1}$, Yan $\mathrm{He}^{1}$, Jun $\mathrm{An}^{1}$, Qiang Miao ${ }^{1}$, Ruo-Xuan Sui ${ }^{1}$, Qing Wang ${ }^{1}$, \\ Jie-Zhong $\mathrm{Yu}^{2}$, Bao-Guo Xiao ${ }^{3 *}$ and Cun-Gen $\mathrm{Ma}^{1,2 *}$ \\ ${ }^{1}$ The Key Research Laboratory of Benefiting Qi for Acting Blood Circulation Method to Treat Multiple Sclerosis of State \\ Administration of Traditional Chinese Medicine, Research Center of Neurobiology, Shanxi University of Chinese Medicine, \\ Taiyuan, China, ${ }^{2}$ Shanxi Key Laboratory of Inflammatory Neurodegenerative Diseases, Institute of Brain Science, Shanxi \\ Datong University, Datong, China, ${ }^{3}$ Institute of Neurology, Huashan Hospital, Institutes of Brain Science and State Key \\ Laboratory of Medical Neurobiology, Fudan University, Shanghai, China
}

Multiple sclerosis (MS) is an inflammatory demyelinating disorder in the central nervous system (CNS), in which remyelination failure results in persistent neurologic impairment. Ginkgolide B (GB), a major terpene lactone and active component of Ginkgo biloba, has neuroprotective effects in several models of neurological diseases. Here, our results show, by using an in vivo cuprizone (CPZ)-induced demyelinating model, administration of GB improved behavior abnormalities, promoted myelin generation, and significantly regulated the dynamic balance of microglia and astrocytes by inhibiting the expression of TLR4, NF-kB and iNOS as well as IL-1 $\beta$ and TNF- $\alpha$, and up-regulating the expression of Arg-1 and neurotrophic factors. GB treatment also induced the generation of oligodendrocyte precursor cells (OPCs). In vitro cell experiments yielded the results similar to those of the in vivo model. The dynamic balance by decreasing microglia-mediated neuroinflammation and promoting astrocyte-derived neurotrophic factors should contribute to endogenous remyelination. Despite GB treatment may represent a novel strategy for promoting myelin recovery, the precise mechanism of GB targeting microglia and astrocytes remains to be further explored.

Keywords: Ginkgolide B, cuprizone-induced demyelination, microglia, astrocytes, remyelination

\section{INTRODUCTION}

Multiple sclerosis (MS) is an inflammatory demyelinating disease of the central nervous system (CNS), accompanied by the following pathological manifestations, such as immune cell invasion, demyelination and axonal degeneration (Reich et al., 2018). Although the exact cause of oligodendrocyte damage and loss is not fully elucidated, several hypotheses have been proposed and are being tested, including oxidative stress, mitochondrial dysfunction, neuroinflammation, protein misfolding (Dendrou et al., 2015; Stone and Lin, 2015; Ibitoye et al., 2016; Rajda et al., 2017).

Abbreviations: MS, multiple sclerosis; CNS, central nervous system; OPCs, oligodendrocyte precursor cells; GB, Ginkgolide B; $\mathrm{RT}$, room temperature. 
In recent years, there has been a paradigm shift in the treatment of MS because of the approval of some new diseasemodifying drugs distinct mechanisms of action (Vaughn et al., 2018). However, the emergence of highly effective treatment options for MS has been accompanied by an increasingly complex array of adverse effects, especially with regard to immune compromise following long-term immune therapy (Klotz et al., 2019). It was reported that Fingolimod is also associated with an increased incidence of other skin malignancies (Velter et al., 2019). Graves' disease is one of the most frequent presentations when Alemtuzumab is effective in the treatment of relapsing-remitting MS (Alamo et al., 2019). The increasing complexity of MS therapies, especially with regard to treatment sequencing and potential additive immune compromise, heightens the need for more effective and safer therapeutic drugs. In addition, axons and neurons are mostly preserved in early MS, leading to gradual neuroaxonal loss correlating with patient disability (Granberg et al., 2017). The results of recent developments in imaging technology have demonstrated that the neurodegenerative process then gradually becomes self-perpetuating, resulting in irreversible disability (Friese et al., 2014). New therapeutic strategies targeting neuroprotection and remyelination are two unmet needs in the treatment of MS (Thompson et al., 2018).

Ginkgo biloba, a slow-growing tree indigenous to Eastern Asia, is one of the oldest species of trees on the planet, with neuroprotective effects (Ahlemeyer and Krieglstein, 2003). In vitro, the extract of Ginkgo biloba protected cultured neurons against death induced by hypoxia, hydrogen peroxide, glutamate, amyloid, nitric oxide, and 1-methyl-4-phenyl-1,2,3,6tetrahydropyridine (MPTP; Ahlemeyer and Krieglstein, 2003). In vivo, the extract of Ginkgo biloba also protected neuron damage and/or death through different mechanisms in middle cerebral artery occlusion (MCAO), focal cerebral ischemia, hypoxia, and amyotrophic lateral sclerosis (ALS) mice or rat models (Ahlemeyer and Krieglstein, 2003). Ginkgolide B (GB), a major terpene lactone and active component of Ginkgo biloba, has neuroprotective effects in several models of neurological diseases. GB alleviated hypoxia-induced neuronal damage in the rat hippocampus by inhibiting oxidative stress and apoptosis (Li et al., 2019) and NLRP3 inflammasome activation (Chen et al., 2018). GB promoted the differentiation of neural stem cells following cerebral ischemia/reperfusion injury (Zheng et al., 2018). Ginkgo biloba extract EGb 761 elicited protective protein clearance through the autophagy-lysosomal pathway in tau-transgenic mice and cultured neurons (Qin et al., 2018). However, there is no experimental information on the therapeutic value of GB for the protection and regeneration of myelin sheaths in the CNS.

CPZ-induced demyelination, unlike experimental autoimmune encephalomyelitis (EAE), is independent of autoimmune attacks and is also often used to mimic the pathology of human MS (Zhen et al., 2017; Vega-Riquer et al., 2019). Remarkably, some events and aspects of the histological pattern induced by $\mathrm{CPZ}$ are similar to those found in MS. It is disappointing that a large number of MS patients suffer from the influence of demyelination such as disable and anxiety in spite of the myelin repairing following with demyelination frequently. Therefore, CPZ-induced demyelination provides a good experimental approach to study demyelination and remyelination and is a suitable pharmacological model for developing some promising drugs of neuroprotection and/or remyelination. In this study, we observed the therapeutic potential of GB for myelin protection and regeneration in the CPZ-induced demyelination model and explored the possible cellular and molecular mechanisms of action.

\section{MATERIALS AND METHODS}

\section{Animals}

Ten-week-old C57BL/6 male mice, weighing 15-20 g, were purchased from Vital River Laboratory Animal Technology Company Limited (Beijing, China) and used for all experiments. Upon arrival, mice were acclimatized and fed for 7 days before starting the experiment. Mice were kept under controlled temperature $\left(25^{\circ} \mathrm{C}\right)$ and humidity with normal light/dark cycle conditions throughout the whole experiment. All animal procedures were approved by the Council for Laboratory and Ethics Committee of Shanxi University of Chinese Medicine, Taiyuan, China. All animal protocol was performed according to the International Council for Laboratory Animal Science guidelines.

\section{CPZ-Induced Demyelinating Model and Administration of GB}

The experimental mice were randomly divided into three groups: normal diet group (Normal, $n=8)$, CPZ diet group (CPZ, $n=8$ ) and $\mathrm{CPZ}$ diet plus $\mathrm{GB}$ intervention group $(\mathrm{CPZ}+\mathrm{GB}, n=7)$. To induce demyelination, mice included in normal and $\mathrm{CPZ}$ groups were fed with $0.2 \%$ (w/w) cuprizone (CPZ; Sigma-Aldrich, USA) in chow diet ad libitum for a total of 6 weeks. After 4 weeks, experimental mice were intraperitoneally (i.p.) injected with GB $(20 \mathrm{mg} / \mathrm{kg}$ ) or normal saline (NS) for consecutive 14 days. One mouse in the $\mathrm{CPZ}+\mathrm{GB}$ group died of unknown causes on the 7 th day after feeding CPZ. No other adverse events occurred in this study.

\section{Behavior Test}

It has been reported that demyelinating lesions are indicative of anxiety-and depression-like behavior and cognitive impairment. Therefore, forced swimming (FS), elevated plus maze (EPM), and T-maze (TM) tests were performed for anxiety, depression and cognitive impairment on the day before the end of the experiment. All behavioral tests were repeated three times in a separate cohort of mice. For EPM, the mice were placed individually in the center of the plus-maze facing an open arm. The number of entering closed arms was recorded during the 10 -min testing period. Distance in the open arm and the number of entries into the open arm were recorded. For FST, the mice were placed individually to swim in a plastic cylinder (height: $30 \mathrm{~cm}$, diameter: $10 \mathrm{~cm}$ ) filled with $20 \mathrm{~cm}$ of $25 \pm 1^{\circ} \mathrm{C}$ water. Cumulative activity distance and total resting time were recorded during $1 \mathrm{~min}$. The TM consisted of two arms and one stem. There was a start box on the bottom of the stem of the maze. 


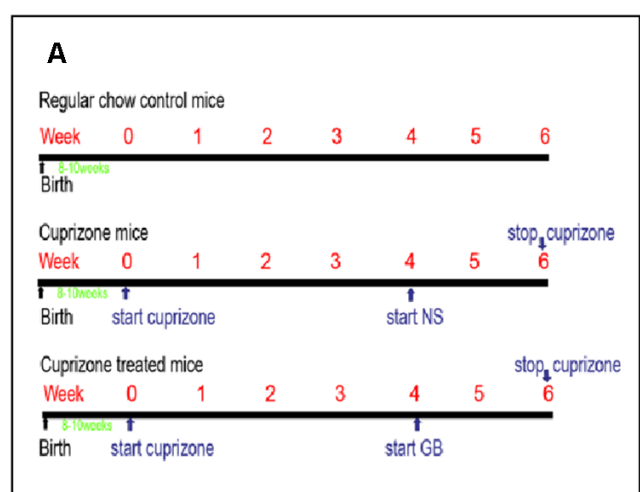

B
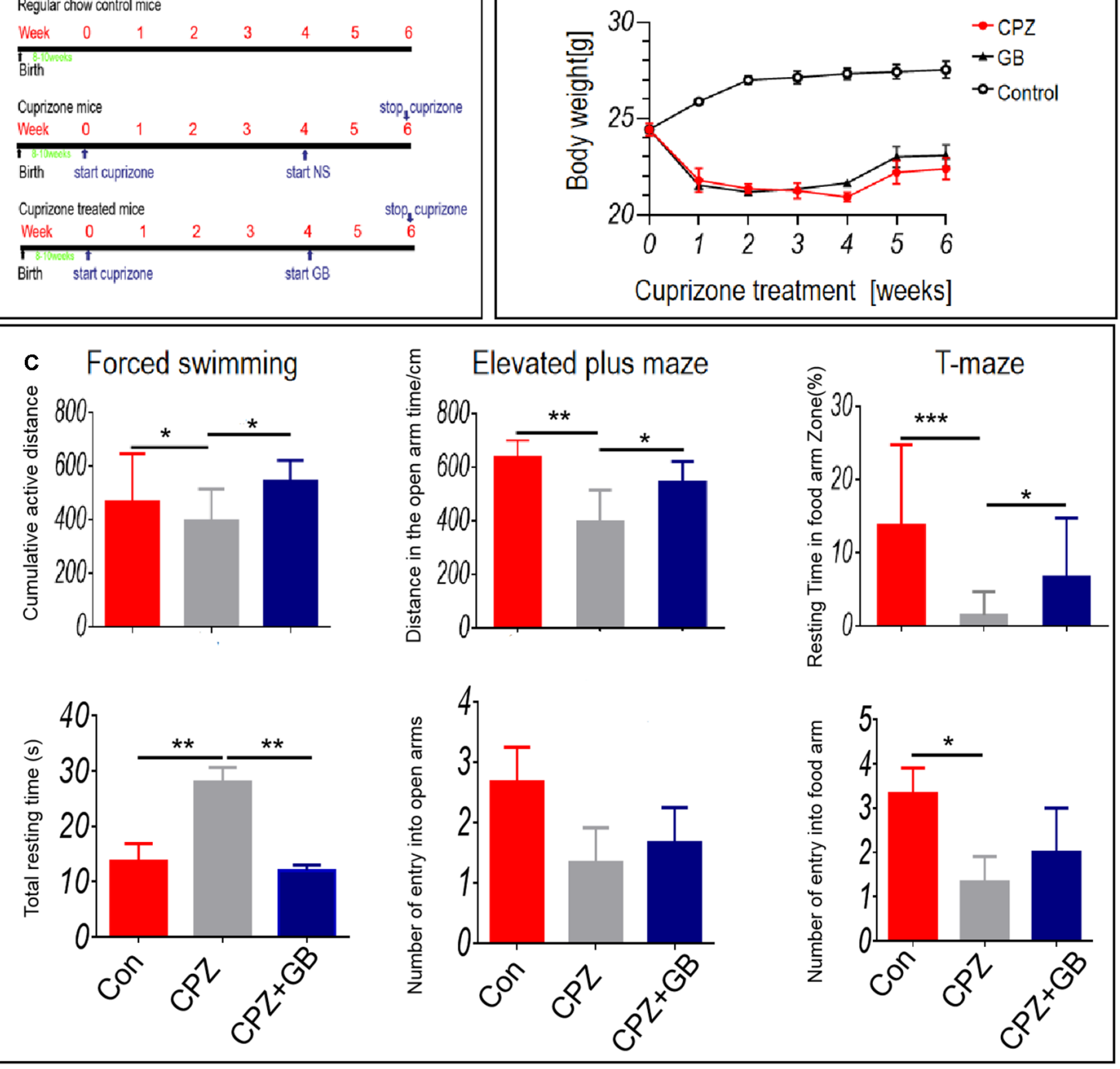

FIGURE 1 | Ginkgolide B (GB) improved behavioral abnormality in cuprizone (CPZ)-induced demyelination. Mice were fed with chow containing 0.2\% CPZ for 6 weeks and were intraperitoneally injected with GB for 14 consecutive days from the 4th to 6th week of CPZ feeding. (A) The design scheme of experimental protocol ( $n=8 /$ group), (B) body weight change, (C) anxiety-and depression-like behavior and cognitive function by forced swimming test (FST), elevated plus maze (EPM) and T-maze (TM) tests. Behavioral results were obtained by video camera and quantified by Image-Pro Plus 6.0 software. The results represent the mean \pm SEM. ${ }^{*} p<0.05,{ }^{* *} p<0.01,{ }^{* * *} p<0.001$

Two target compartments were located at the end of both arms of the maze. Mice were tested 10 times per day for 3 days. Mice were positioned at the end of one stem and given the possibility to move for $10 \mathrm{~min}$. Resting time in food arm zone and number of entry into food arm were recorded. All data acquisition and analysis were performed automatically using digital video and Image $^{\mathrm{TM}}$ software.

\section{Tissue Preparation}

After saline infusion and fixation with $10 \%$ chloralhydrate, the brain ( $n=3-4)$ was carefully removed, immersed in $30 \%$ sucrose solution for $24 \mathrm{~h}$, and then stored at $-80^{\circ} \mathrm{C}$ for subsequent immunohistochemistry. The other half of the mice $(n=4)$ only received a saline infusion, and the brain was removed and stored at $-80^{\circ} \mathrm{C}$ for subsequent enzyme-linked immunosorbent assay (ELISA) and western blot assays.

\section{Myelin Staining}

Histological myelin staining was performed by Luxol Fast Blue (LFB) staining and Black Gold II staining. LFB staining: the slides were stained in $\mathrm{LFB}$ at $56^{\circ} \mathrm{C}$ overnight. After washing with $95 \%$ ethanol and distilled water, the color was differentiated in lithium carbonate solution for $15 \mathrm{~s}$ followed by distilled water and three washes of $80 \%$ alcohol. Black Gold II staining: the slides were 

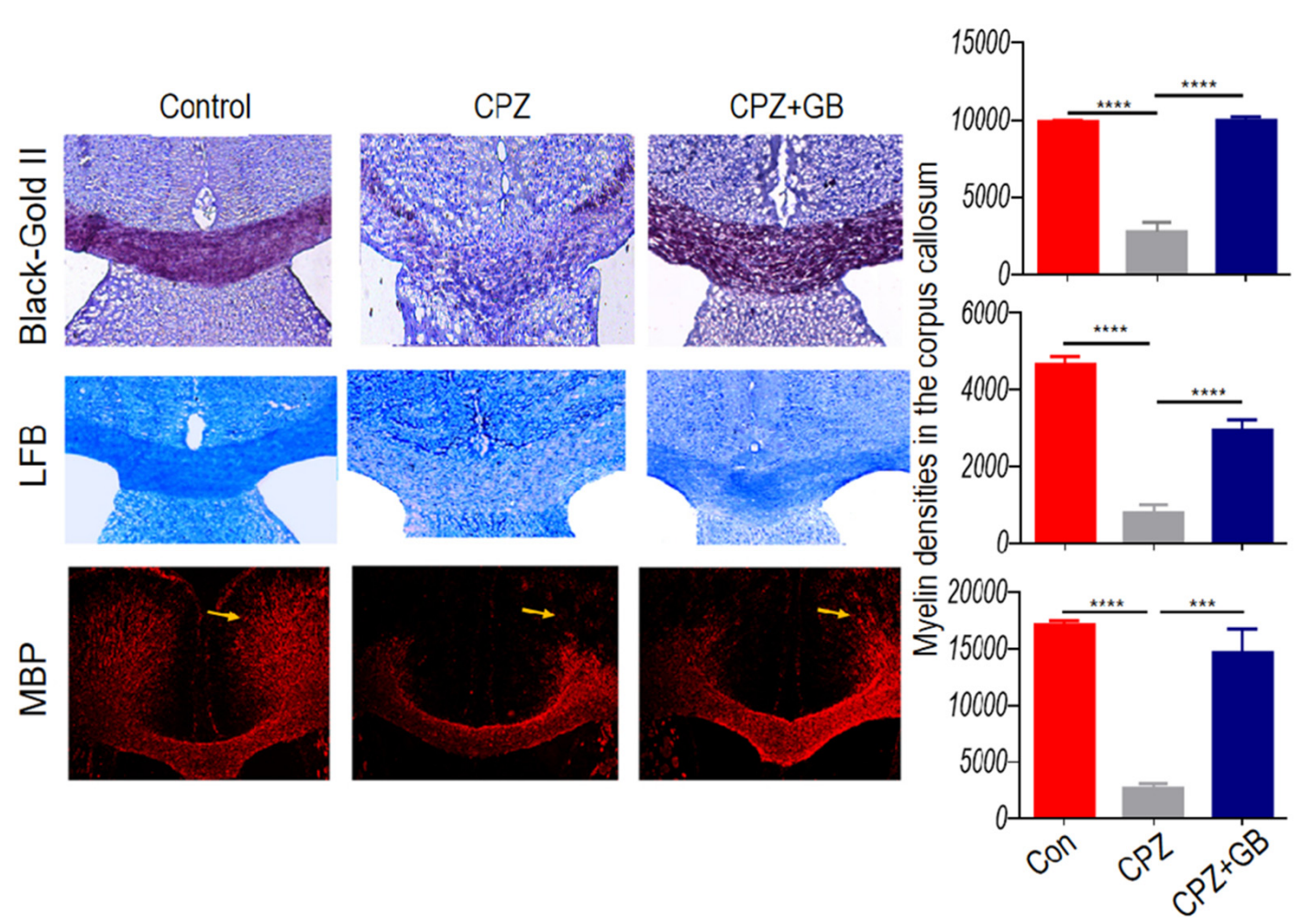

FIGURE 2 | GB promoted remyelination in CPZ-induced demyelination. Mice were fed with chow containing $0.2 \%$ CPZ for 6 weeks and were intraperitoneally injected with GB for 14 consecutive days from the 4th to 6th week of CPZ feeding. Histological evaluation of demyelination was carried out by Black Gold II, luxol fast blue (LFB) and myelin basic protein (MBP) staining ( $n=3-4$ /each group), and quantified by Image-Pro Plus 6.0 software. Representative images of Black Gold II, LFB and MBP staining are observed in the corpus callosum of the brain. The results represent the mean \pm SEM. ${ }^{* * *} p<0.001,{ }^{* * * *} p<0.0001$.

dehydrated for 60 min on a slide warmer and then rehydrated with purified water. Pre-warmed Black Gold II solution was added onto sections and incubated at $60^{\circ} \mathrm{C}$ for $15 \mathrm{~min}$. After washing with Milli-Q water, pre-warmed $1 \%$ sodium thiosulfate was added to the slides and incubated for $3 \mathrm{~min}$, followed by the incubation with cresyl violet stain for $3 \mathrm{~min}$. The mean optical densities of LFB and Black Gold II staining in the corpus callosum were measured using Image-Pro Plus 6.0 software. Myelin basic protein (MBP) staining: after blocking with $1 \%$ $\mathrm{BSA} / \mathrm{PBS}$ at room temperature (RT) for $30 \mathrm{~min}$, the slides were incubated with anti-MBP (1:500, Abcam, Burlingame, CA, USA) at $4^{\circ} \mathrm{C}$ for $18 \mathrm{~h}$, and then incubated with anti-rabbit IgG (1:1,000, Abcam, Burlingame, CA, USA) at RT for $2 \mathrm{~h}$. As a negative control, additional sections were treated similarly, but the primary antibodies were omitted. Results were visualized and analyzed under fluorescent microscopy by Image-Pro Plus 6.0 software described by immunohistochemistry in a blinded fashion. Quantification was performed on three sections per mouse.

\section{Immunohistochemistry}

Brain slides were blocked with $1 \%$ BSA/PBS for $1 \mathrm{~h}$ at RT and incubated with anti-MBP (1:500, Abcam, Burlingame, CA, USA), anti-Iba-1 (1:300, BD Bioscience, USA), anti-NFкB/p65 (1:200, Cell Signaling Technology, Danvers, MA, USA),
anti-TLR4 (1:200, Bioworld Tech. Inc., St. Louis Park, MN, USA), anti-iNOS (1:300, Abcam, Burlingame, CA, USA), antiArg-1 (1:300, Gene Tex, Irvine, CA, USA), anti-GFAP (1:250, Thermo Fisher, USA), anti-BDNF (1:200, Novusbio, Centennial, CO, USA), anti-GDNF (1:500, Abcam, Burlingame, CA, USA), anti-NG2 (1:300, Millipore, Germany) and anti-Ki67 (1:200, BD Pharmingen, USA) at $4^{\circ} \mathrm{C}$ for overnight, followed by corresponding secondary antibodies at RT for $1 \mathrm{~h}$. The results were repeated three times with consecutive brain slices from each group, and the slides were observed under fluorescence microscopy in a blinded fashion. Analysis and quantification were done in three sections/per mouse by Image-Pro Plus 6.0 software.

\section{Western Blot Analysis}

Extracts of protein $(30 \mu \mathrm{g})$ were separated by sodium dodecyl sulfate-polyacrylamide gel electrophoresis (SDS-PAGE) and subsequently transferred electrophoretically to nitrocellulose membrane (Millipore). For SDS-PAGF, 10\% separating gel and 5\% stacking gel were used to perform electrophoretic separation. After blocking with 5\% skimmed milk at RT for $1 \mathrm{~h}$, the membranes were incubated with rabbit antiIba-1 (1:1,000, Abcam, Burlingame, CA, USA), rabbit antiTLR4(1:500, Abcam, Burlingame, CA, USA), rabbit anti-NFKB (1:800, Abcam, Burlingame, CA, USA), rabbit anti-iNOS (1:800, 

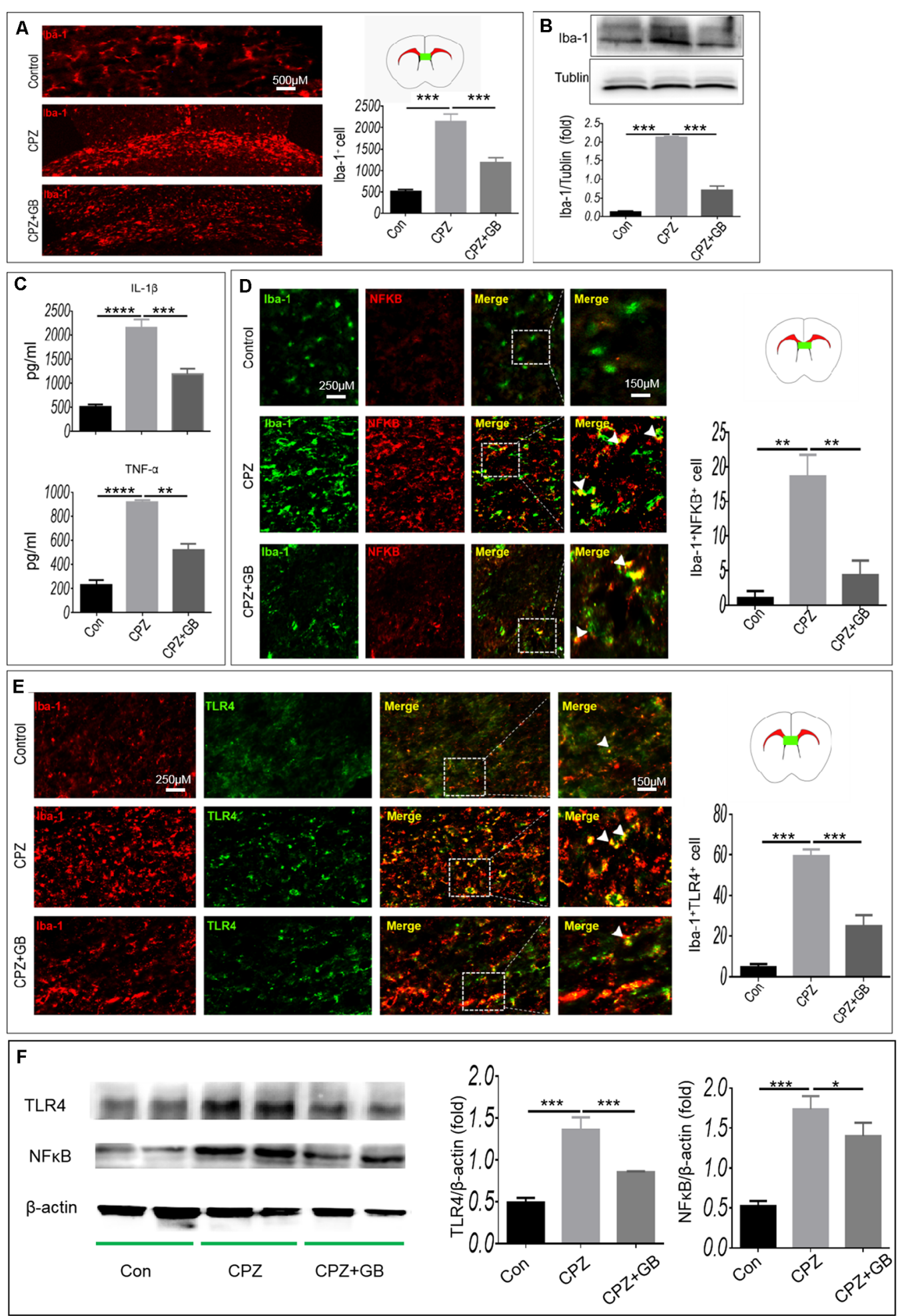

FIGURE 3 | GB inhibited the microglial inflammatory response. Mice were fed with chow containing $0.2 \% \mathrm{CPZ}$ for 6 weeks and were intraperitoneally injected with GB for 14 consecutive days from the 4th to 6 th week of CPZ feeding. (A) lba- $1^{+}$microglia in the corpus callosum of brain by immunohistochemistry, (B) expression of Iba- $1^{+}$protein in the extract of brain by western blot, (C) levels of inflammatory cytokines IL-1 $\beta$ and TNF- $\alpha$ in the extract of brain by enzyme-linked immunosorbent assay (ELISA), (D) double immunohistochemical staining with anti-lba-1 and anti-p-NF-kB/p65 in the corpus callosum, (E) double immunohistochemical staining with anti-lba-1 and anti-TLR4 in the corpus callosum. (F) Expression of TLR4 and p-NF-kB/p65 in the extract of the brain by western blot. Representative images are obtained from three to four mice/each group, with similar results. The results represent the mean \pm SEM. ${ }^{*} p<0.05,{ }^{* *} p<0.01,{ }^{* * *} p<0.001,{ }^{* * * *} p<0.0001$. 

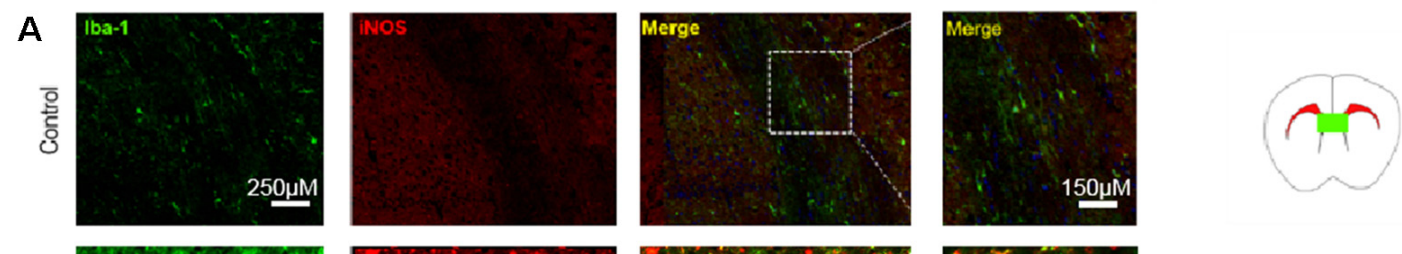

壳
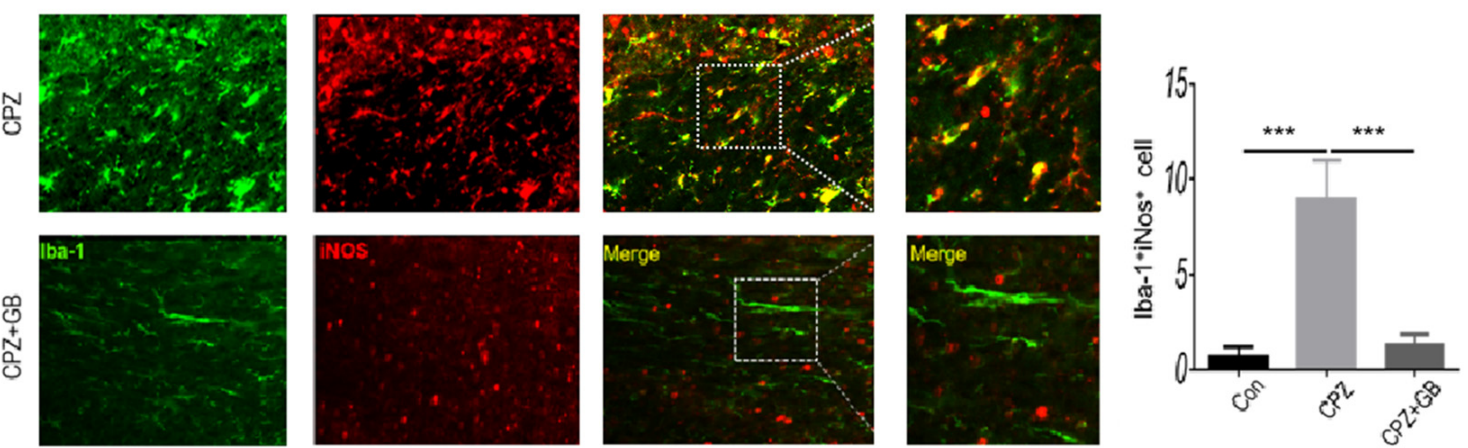

B
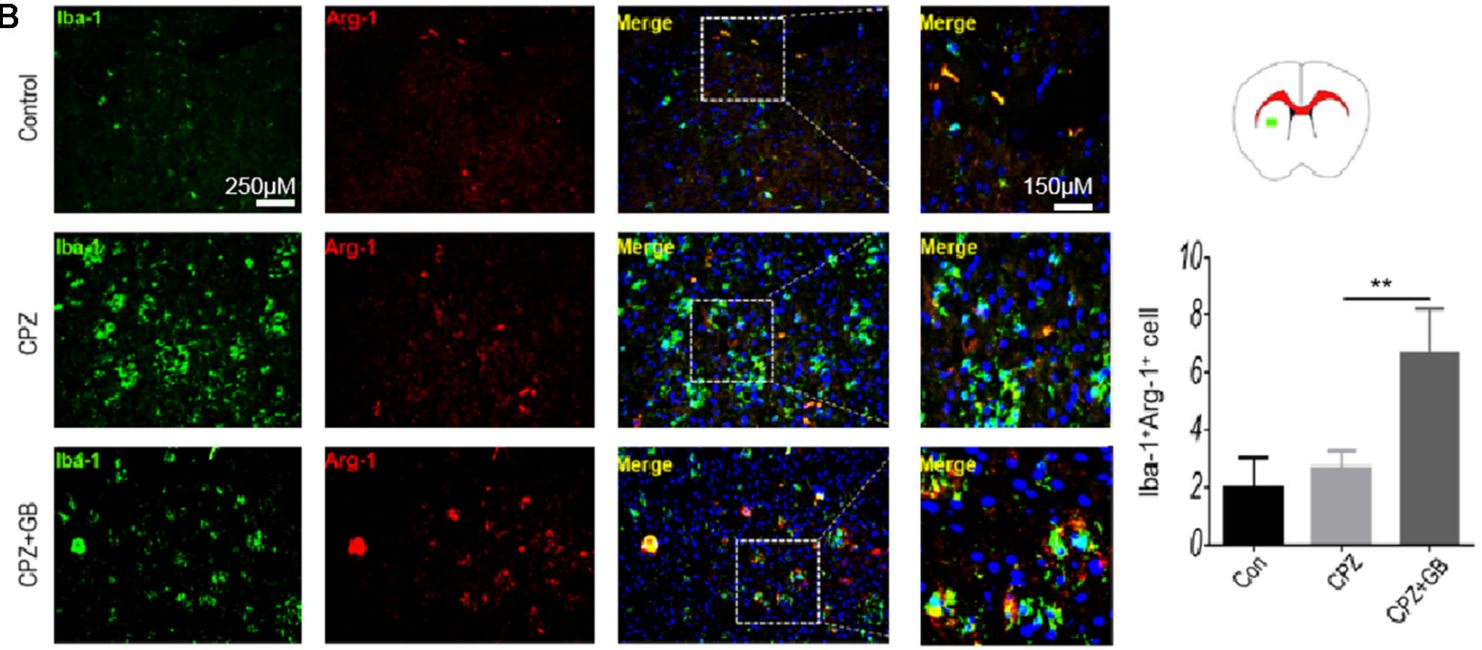

C
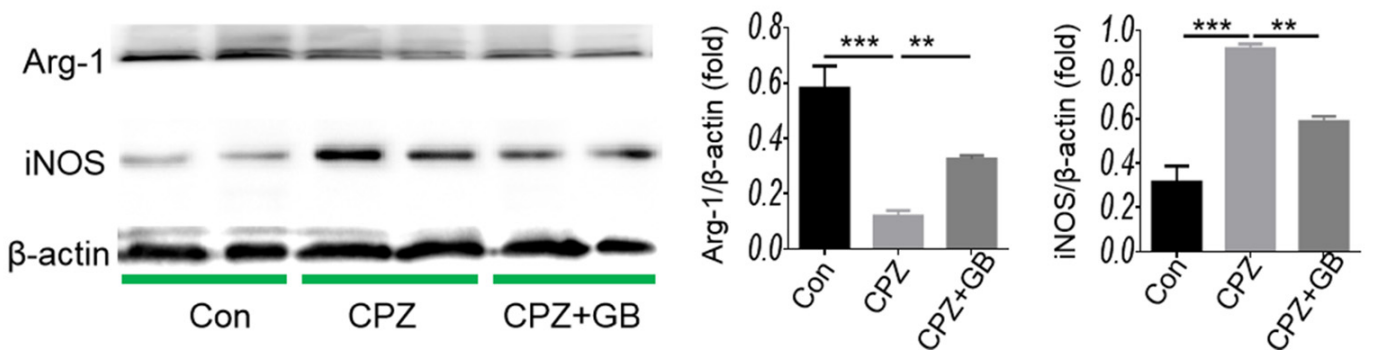

FIGURE 4 | GB influenced the phenotype of M1/M2 microglia. Mice were fed with chow containing $0.2 \%$ CPZ for 6 weeks and were intraperitoneally injected with GB for 14 consecutive days from the 4th to 6th week of CPZ feeding. (A) Double immunohistochemical staining with anti-lba-1 and anti-iNOS in the corpus callosum, (B) double immunohistochemical staining with anti-lba-1 and anti-Arg-1 in the striatum. (C) Expression of Arg-1 and iNOS protein in the extract of the brain by western blot. Representative images are obtained from three to four mice/each group, with similar results. The results represent the mean \pm SEM. ${ }^{* *} p<0.01,{ }^{* * *} p<0.001$.

Gene Tex, Irvine, CA, USA), rabbit anti-Arginase 1 (1:500, Gene Tex, Irvine, CA, USA), rabbit anti-GFAP (1:1,000, Thermo Fisher, USA), rabbit anti-tubulin (1:5,000, Bioword, USA) and rabbit anti- $\beta$-actin $(1: 1,000$, Cell Signaling Technology,
USA) at $4^{\circ} \mathrm{C}$ for overnight, followed by HRP-conjugated goat anti-rabbit secondary antibodies (1:1,000, Abcam, Burlingame, CA, USA) at RT for $2 \mathrm{~h}$. Immunoblots were developed with an enhanced chemiluminescence system (GE Healthcare 


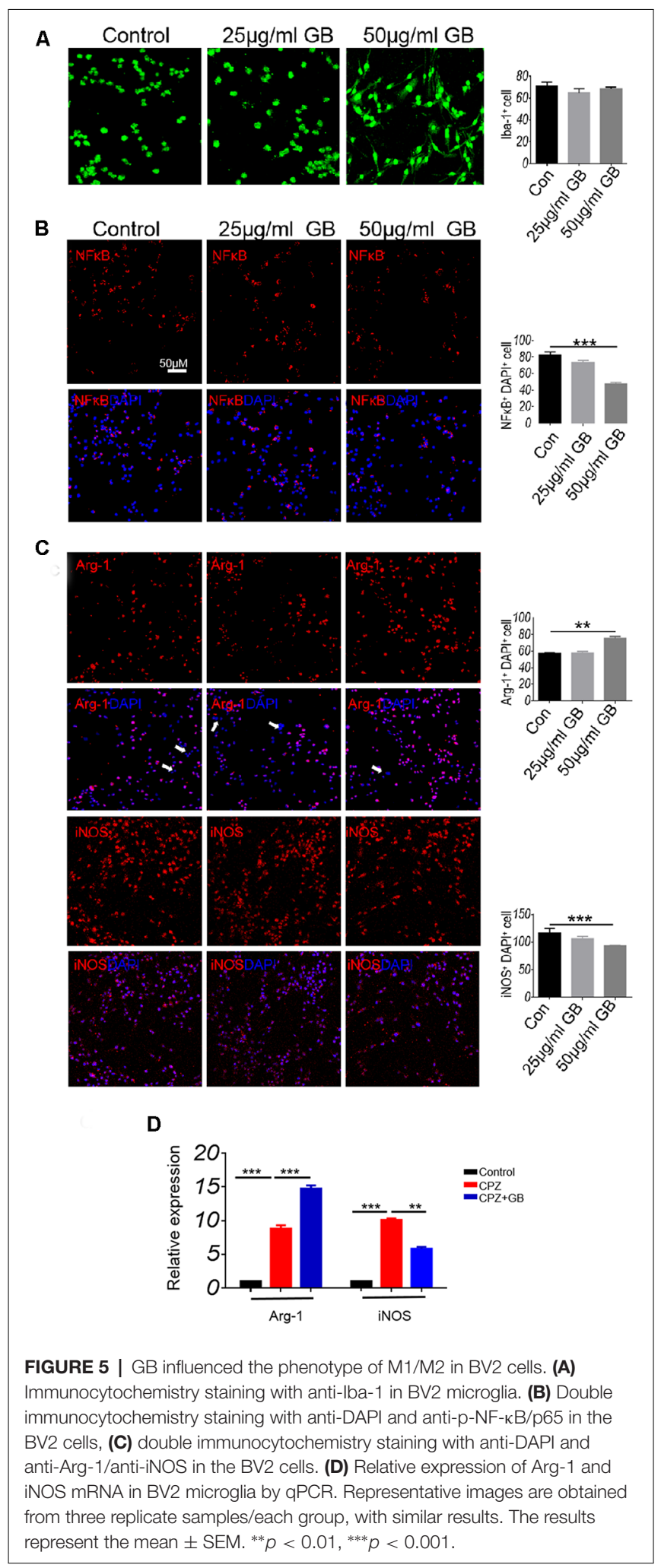

Life Sciences) and measured using Quantity Software (BioRad, Hercules, CA, USA). Tublin and $\beta$-actin were used as an internal reference. The experiment was repeated at least three times.

\section{Enzyme-Linked Immunosorbent Assay (ELISA)}

The concentrations of IFN- $\gamma$, TNF- $\alpha$, BDNF, GDNF in brain homogenates were measured by sandwich ELISA kit (R\&D system, USA) according to the manufacturer's instructions. ELISA procedure should be done at RT but the standard dilutions kept on ice while during preparation. We use $\mathrm{pg} / \mathrm{ml}$ to express the results.

\section{Cell Culture}

\section{Astrocytes}

Primary astrocytes were prepared from postnatal (1-day-old) C57BL/6 mice. Under aseptic conditions, cerebral cortices were dissected and placed in sterile phenol red-free Dulbecco's Modified Eagle's Medium (DMEM, 4,500 mg/l Glucose, 4 mM L-Glutamine, $1 \mathrm{mM}$ Sodium Pyruvate, Thermo Scientific) containing $1 \%$ of streptomycin $(10,000 \mu \mathrm{g} / \mathrm{ml})$-penicillin $(10,000$ units $/ \mathrm{ml})$. The cells were dissociated into a single cell suspension with a Pasteur pipette, and then seeded in 6-well culture plates at a density of $2.5 \times 10^{5}$ cells/well in a humidified incubator at $37^{\circ} \mathrm{C}$ and $5 \% \mathrm{CO}_{2}$. When mixed glial cultures reached $90 \%$ confluence (typically 8-10 days), the microglia in the cultures were removed by shaking constantly for 4-6 h. Immunocytochemical staining and quantitative PCR were measured by using cultured primary astrocytes.

\section{Microglia}

The BV2 mouse microglia cell line (from ShenKe Biological Technology Company Limited, Shanghai, China) was cultured in Dulbecco's modified Eagle medium (DMEM, Gibco, Gaithersburg, MD, USA) with 10\% fetal bovine serum, $100 \mu / \mathrm{ml}$ penicillin, and $100 \mathrm{mg} / \mathrm{ml}$ streptomycin (complete medium) at $37^{\circ} \mathrm{C}$ and a humidified atmosphere augmented with $5 \% \mathrm{CO}_{2}$. Cells were plated at a density of $1 \times 10^{5} / \mathrm{ml}$ for all experiments. The BV2 was stimulated with LPS at a concentration of $1 \mu \mathrm{g} / \mathrm{ml}$ and the stimulation time was $12 \mathrm{~h}$.

\section{Oligodendrocytes}

Oligodendrocytes were isolated from the brain of newborn mice (1-day-old) by CD140a MicroBead kit (Miltenyi Biotec) according to product instructions. Cells were plated at a density of $1 \times 10^{5} / \mathrm{ml}$ for all experiments. PDGFR $\alpha$ was expressed in sorted cells.

\section{Immunocytochemistry}

Cells were blocked with $1 \%$ BSA/PBS for $1 \mathrm{~h}$ at RT and incubated with anti-Iba-1 (1:300, BD Bioscience, San Jose, CA, USA), anti-NF- $\kappa$ B/p65 (1:200, Cell Signaling Technology, USA), anti-iNOS (1:300, Abcam, Burlingame, CA, USA), anti-Arg-1 (1:300, Gene Tex, Irvine, CA, USA), anti-GFAP (1:250, Thermo Fisher, USA), anti-BDNF (1:200, Novusbio, Centennial, CO, USA), anti-GDNF (1:500, Abcam, USA), anti-PDGFR $\alpha$ (1:300, Millipore, Germany) and anti-Ki67 (1:200, BD Pharmingen, USA) at $4^{\circ} \mathrm{C}$ for overnight, followed by corresponding secondary antibodies at RT for $1 \mathrm{~h}$. The results were repeated three times with similar results. Analysis and quantification were done by Image-Pro Plus 6.0 software. 

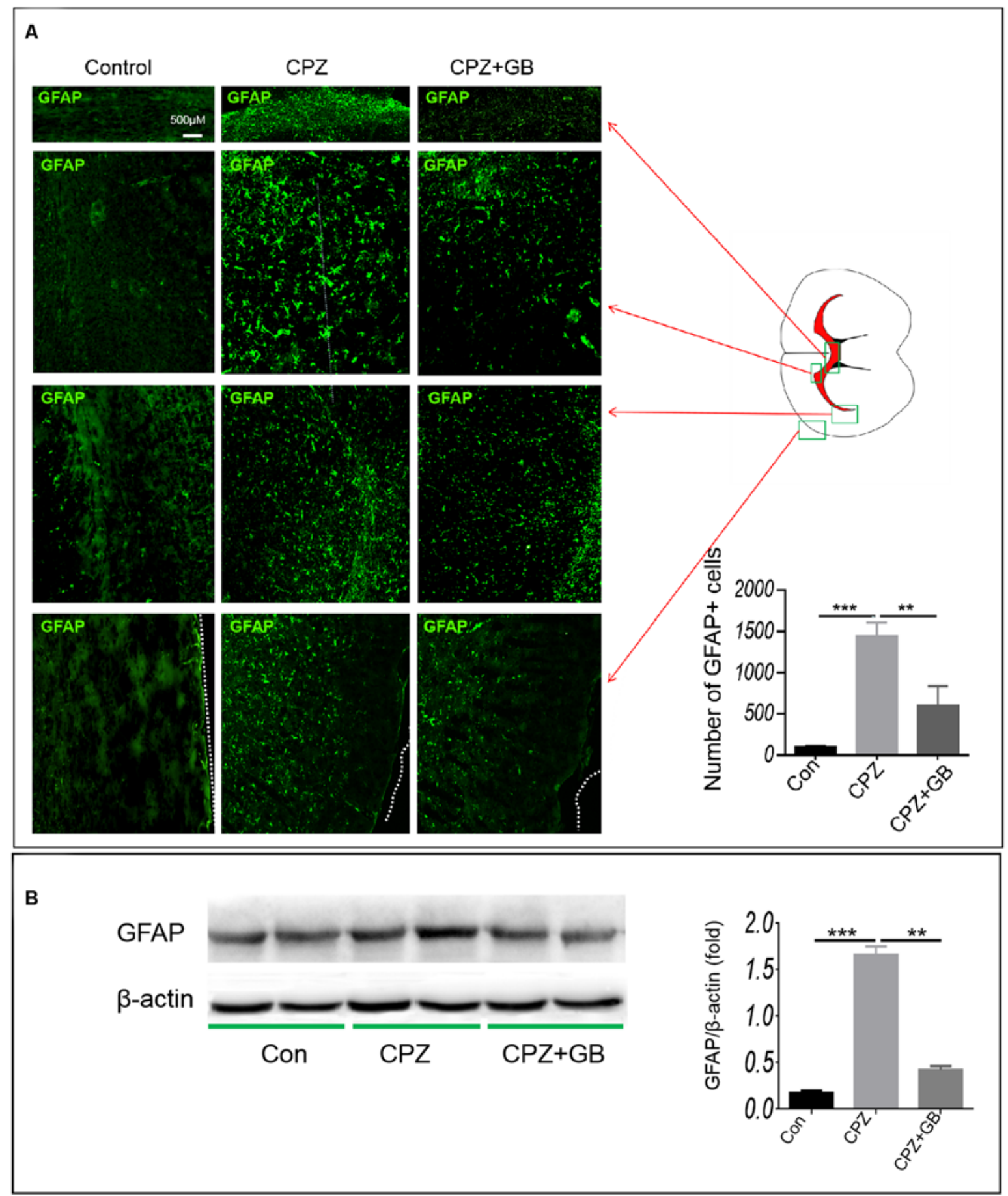

FIGURE 6 | GB inhibited astrocyte response. Mice were fed with chow containing $0.2 \%$ CPZ for 6 weeks and were intraperitoneally injected with GB for 14 consecutive days from the 4th to 6th week of CPZ feeding. (A) Distribution of GFAP ${ }^{+}$astrocytes in the corpus callosum, claustrum, cingulate cortex and cortex of the brain by immunohistochemistry, (B) expression of GFAP protein in the extract of the brain by western blot. Representative images are obtained from three to four mice/each group, with similar results. The results represent the mean \pm SEM. ${ }^{* *} p<0.01,{ }^{* * *} p<0.001$.

\section{Quantitative RT-PCR}

Cells were lysed in Buffer RL, and the total RNA was isolated by RNAprep Pure cell Kit. The total RNA yield and concentration were measured by using Thermo $\left(\mathrm{N}_{\mathrm{ANO}} \mathrm{D}_{\mathrm{ROP}} \mathrm{O}_{\mathrm{NE}}\right)$. cDNA was then generated using the Prime Script ${ }^{\mathrm{TM}}$ RT Master Mix (Takara Bio, Japan), according to the manufacturer's instruction. 


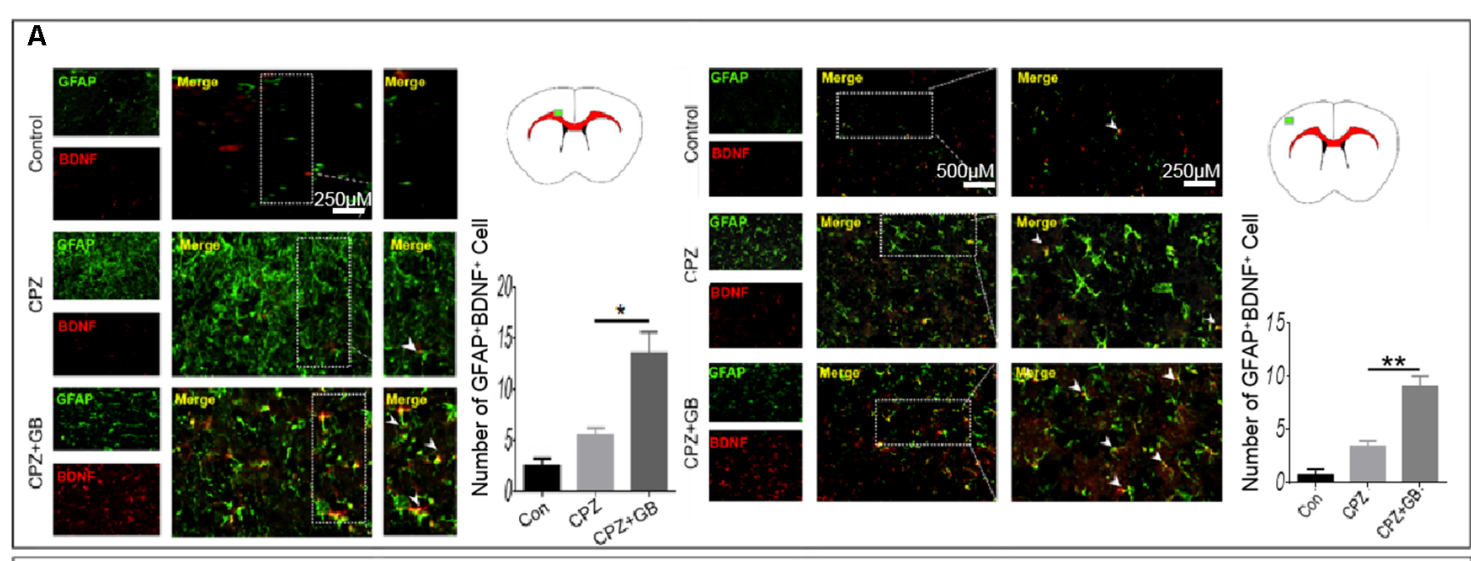

B
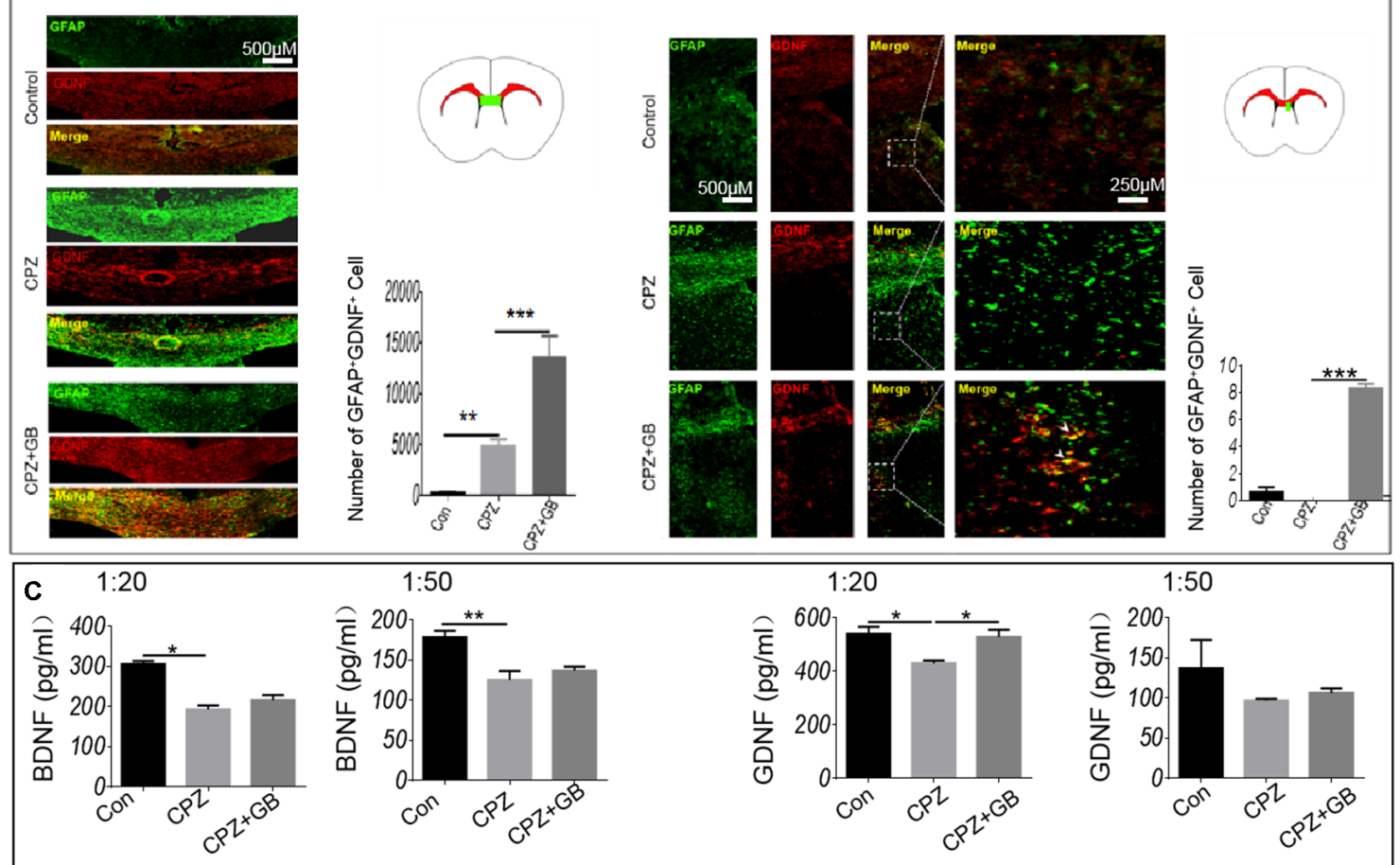

FIGURE 7 | GB induced the production of BDNF and GDNF from astrocytes. Mice were fed with chow containing $0.2 \%$ CPZ for 6 weeks and were intraperitoneally injected with GB for 14 consecutive days from the 4th to 6th week of CPZ feeding. (A) Double immunohistochemical staining with anti-GFAP and anti-BDNF in the cingulate cortex and cortex, (B) double immunohistochemical staining with anti-GFAP and anti-GDNF in the corpus callosum and subventricular region. (C) Levels of BDNF and GDNF in the extract of the brain by ELISA. Representative images are obtained from three to four mice/each group, with similar results. The results represent the mean \pm SEM. ${ }^{*} p<0.05,{ }^{* *} p<0.01,{ }^{* * *} p<0.001$.

Quantitative real-time PCR was performed by using TB Green ${ }^{\mathrm{TM}}$ Premix Ex Taq ${ }^{\mathrm{TM}}$ II (Takara, Japan) on the CFX96 ${ }^{\mathrm{TM}}$ real-time PCR instrument.

The primers used in this study are as follows: BDNF:(FWDAGCTCCTGACCTTGGTCTTG, REV-AGACCTCTCGAACC TGCCC), GDNF:(FWD-AAGTGGCACAGTTTTGCTGGA, RE V-GCTAACAGTGACATCACACAAGT), iNOS:(FWD-CAGG GAGAACAGTACATGAACAC, REV-TTGGATACACTGCTA CAGGGA), Arg-1:(FWD-CTGAGAGATTCAAGGCAAGAGG, REV-GAACGCGCTATCTTACCCCAG), ACTB:(FWD-GTGC
TATGTTGCTCTAGACTTCG, REV-ATGCCACAGGATTCC ATACC).

Cycle conditions were $95^{\circ} \mathrm{C}$ for $30 \mathrm{~s}$, followed by 40 cycles of $95^{\circ} \mathrm{C}$ for $5 \mathrm{~s}$ and $60^{\circ} \mathrm{C}$ for $30 \mathrm{~s}$, and the melt curve was $95^{\circ} \mathrm{C}$ for $15 \mathrm{~s},-60^{\circ} \mathrm{C}$ for $1 \mathrm{~min}$ and $-95^{\circ} \mathrm{C}$ for $15 \mathrm{~s}$.

\section{Statistical Analysis}

Statistical analysis of differences between groups was performed by one-way analysis of variance (ANOVA). All statistical comparisons were performed using GraphPad Prism version 

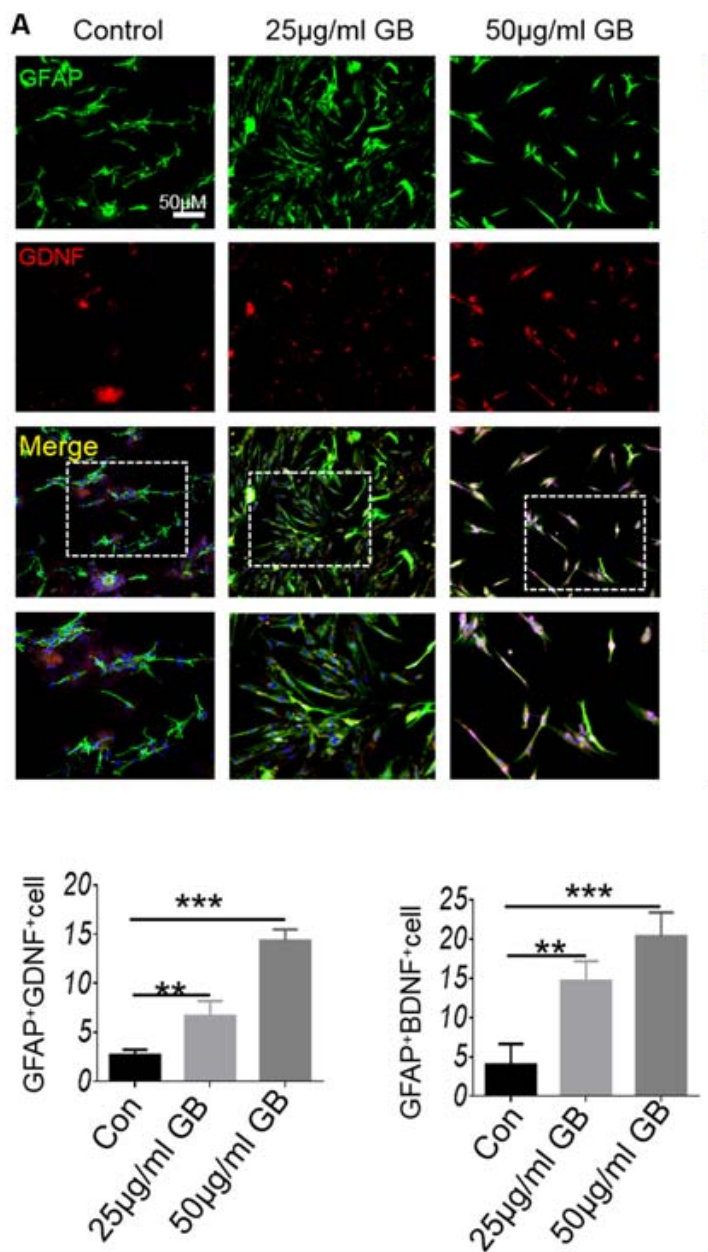
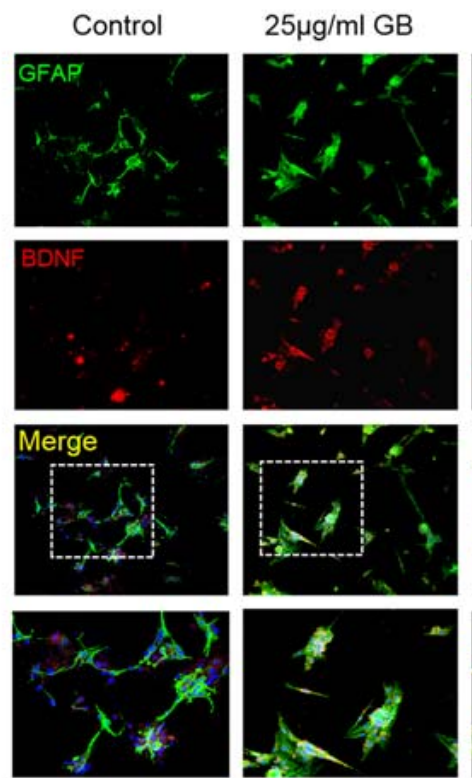

$50 \mu \mathrm{g} / \mathrm{ml} \mathrm{GB}$
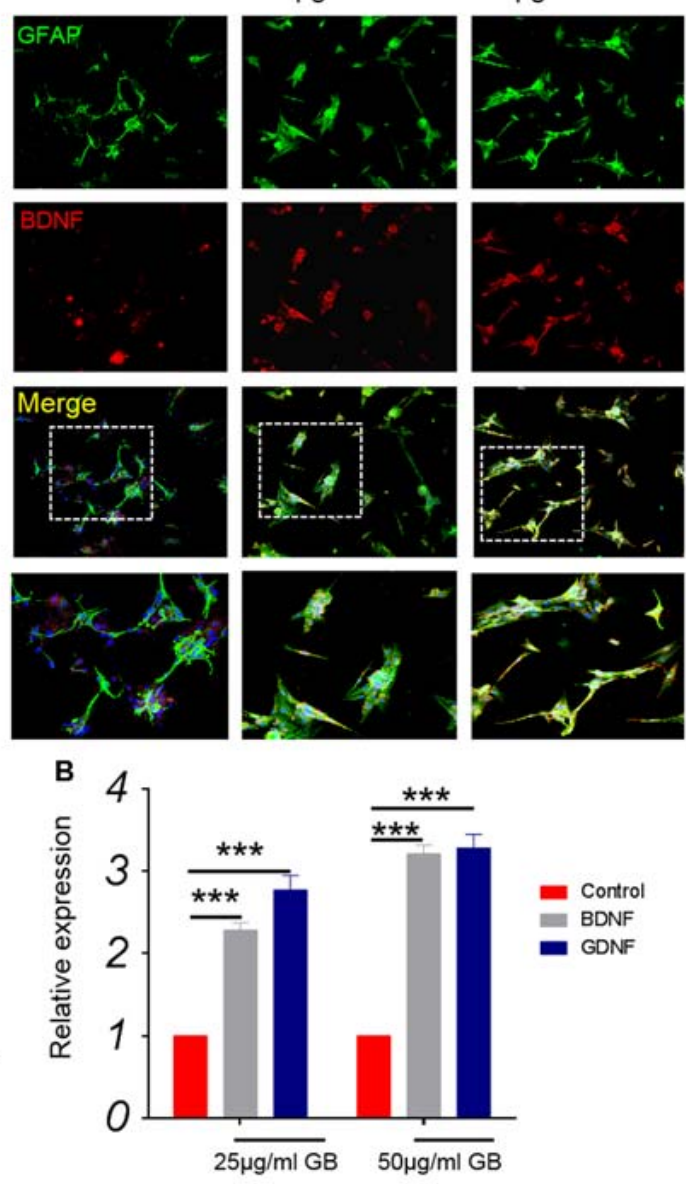

FIGURE 8 | GB promoted the production of neurotrophic BDNF and GDNF in primary astrocytes. (A) Double immunocytochemistry staining with anti-GFAP and anti-BDNF/anti-GDNF in the primary astrocytes. (B) Relative expression of BDNF and GDNF mRNA in the primary astrocytes by qPCR. Representative images are obtained from three replicate samples/each group, with similar results. The results represent the mean $\pm \mathrm{SEM}$. ${ }^{* *} p<0.01,{ }^{* * *} p<0.001$.

6.0 (GraphPad Software, San Diego, CA, USA). All data are expressed as mean \pm SEM. The difference of $p<0.05$ was considered to be statistically significant.

\section{RESULTS}

\section{Ginkgolide B Improves Behavior and Promotes Myelin Repair}

The acute demyelinating model was induced by feeding 6 weeks of C57BL/ 6 mice with a $0.2 \% \mathrm{CPZ}$. The experimental design is shown in Figure 1A. One week after CPZ feeding, the weight of mice fed with $\mathrm{CPZ}$ was significantly decreased than that of mice fed with normal diet (Figure 1B). Although the weight of mice treated with GB was slightly increased than that of $\mathrm{CPZ}$ mice, there was no statistical significance (Figure 1B).

Anxiety- and depression-like behavior and cognitive impairment have been observed in demyelinating lesions
(Acharjee et al., 2013; Xu et al., 2019; Zimmermann et al., 2019). In this study, FS, EPM and TM tests were used to evaluate anxiety-and depression-like behavior and cognitive impairment. The results showed that $\mathrm{CPZ}$ resulted in above behavioral abnormalities after 6 weeks of CPZ feeding as compared with mice fed with normal diet (Figure 1C, $p<0.05, p<0.01$ and $p<0.001$, respectively). However, GB treatment improved effectively these behavioral abnormalities after 2 weeks of treatment (Figure 1C, $p<0.05, p<0.01$, respectively).

To evaluate the protective effect of GB during demyelination, three methods including Black Gold II, LFB, and MBP staining, were simultaneously used in this study. The results showed that compared with normal mice, the myelin sheath in the corpus callosum of mice fed with CPZ was obviously lost by both Black Gold II and LFB staining (Figure 2, $p<0.0001$, respectively). GB treatment effectively increased the intensity of Black Gold II and LFB staining (Figure 2, $p<0.0001$, respectively), suggesting the protective or regenerative role of GB during demyelination. 


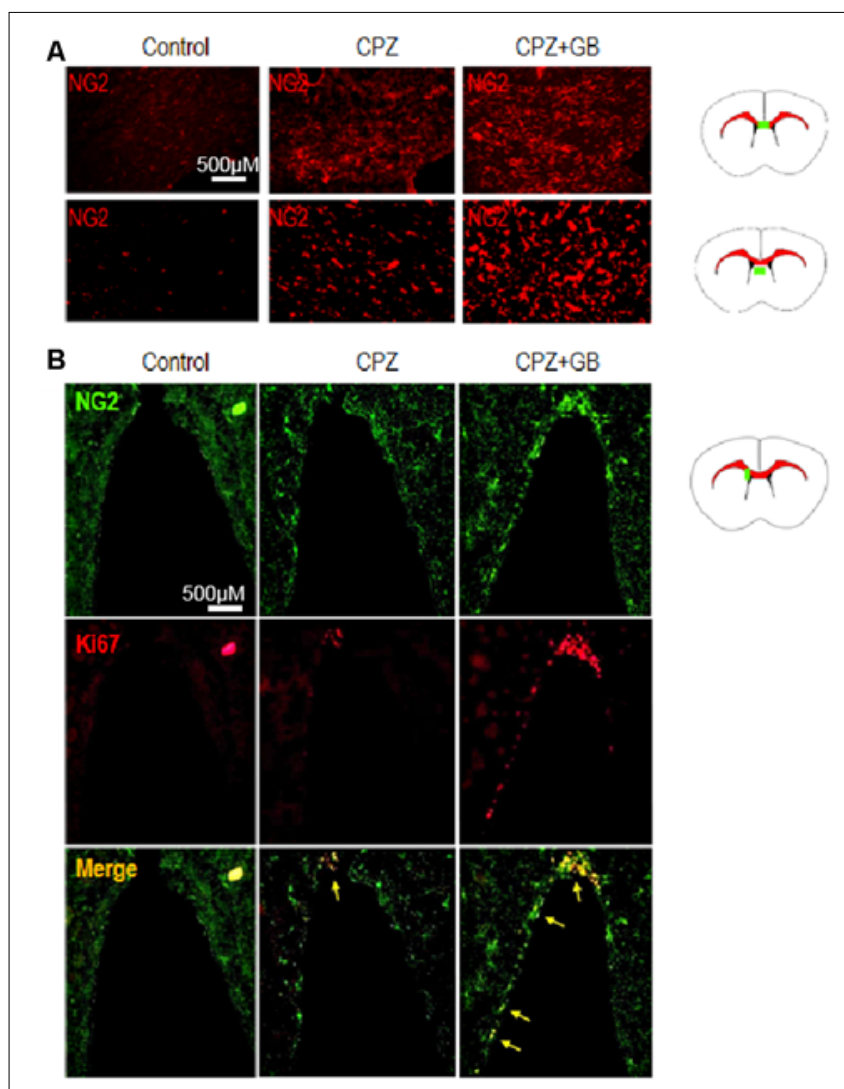

FIGURE 9 | GB promoted the generation of NG2 oligodendrocyte precursor cells (OPCs). Mice were fed with chow containing 0.2\% CPZ for 6 weeks and were intraperitoneally injected with GB for 14 consecutive days from the 4th to 6 th week of $\mathrm{CPZ}$ feeding. (A) NG2 ${ }^{+}$OPCs in the corpus callosum and lateral septal nucleus of the brain by immunohistochemistry, and (B) double immunohistochemical staining with anti-NG2 and anti-Ki67 in the subventricular zone (SVZ; yellow arrow) by immunohistochemistry.

Representative images are obtained from three to four mice/each group, with similar results.

Similarly, MBP staining showed the damage to myelin sheath caused by CPZ feeding (Figure 2, $p<0.0001$ ), which could be repaired by GB treatment, especially in the cingulum region (Figure 2, $p<0.001$ ).

\section{Ginkgolide B Reduces Inflammation Associated With Microglia}

The CPZ model is characterized by primary and reversible demyelination, accompanied by microglia-mediated neuroinflammation that can aggravate demyelinating lesions in the CPZ model. Compared with normal mice, CPZ-fed mice showed the accumulation of $\mathrm{Iba}_{-} \mathrm{1}^{+}$microglia in the corpus callosum (Figure 3A,p<0.001) and the increase of Iba-1 expression in the brain (Figure 3B, $p<0.001$ ), suggesting that myelin damage may trigger the migration and enrichment of microglia in the corpus callosum. However, GB treatment obviously declined the accumulation of $\mathrm{Iba}_{-} 1^{+}$microglia in the corpus callosum and the increase of Iba- 1 expression in the brain (Figures 3A,B, $p<0.001$, respectively).
It is generally believed that $\mathrm{CPZ}$ feeding resulted in the activation of microglia. As shown in Figure $3 \mathrm{C}$, the levels of inflammatory cytokines IL-1 $\beta$ and TNF- $\alpha$ wee elevated in CPZ-fed mice ( $p<0.0001$, respectively), which can be declined by GB treatment $(p<0.001, p<0.0001$, respectively). Next, we observed the phenotypic characteristics of microglia in the corpus callosum. As shown in Figure 3D, the expression of NF- $\mathrm{BB}$ and TLR4 on $\mathrm{Iba}-1^{+}$microglia in CPZ-fed mice was higher than that in normal-fed mice (Figures 3D,E, $p<0.01$ and $p<0.001$, respectively). GB treatment inhibited the expression of NF- $\mathrm{B}$ and TLR4 on $\mathrm{Iba}-1^{+}$microglia in CPZ-fed mice (Figures 3D,E, $p<0.01$ and $p<0.001$, respectively). The results from Western blot showed that the expression of TLR4 and p-NF$\kappa \mathrm{B} / \mathrm{p} 65$ was up-regulated 6 weeks after $\mathrm{CPZ}$ feeding (Figure 3F, $p<0.001$ respectively), and was effectively inhibited by GB intervention (Figure 3F, $p<0.001$ and $p<0.05$, respectively), which is consistent with the that of immunohistochemical staining in Figures 3D,E.

iNOS and Arg-1 are two typical markers of M1 and M2 microglia, respectively. We further used immunohistochemistry to detect the expression of iNOS and Arg-1 on Iba- $1^{+}$microglia. The results showed that Iba- $1^{+}$ microglia expressing iNOS in mice fed with CPZ was significantly higher than that in normal mice (Figure 4A, $p<0.001$ ), which was effectively inhibited by GB treatment (Figure 4A, $p<0.001$ ). Conversely, CPZ feeding did not decline the expression of Arg-1 on Iba- $1^{+}$microglia, but which was indeed up-regulated by GB treatment, as compared with CPZ-fed mice (Figure $4 \mathrm{~B}, p<0.01$ ). The results from Western blot showed that the expression of Arg-1 was inhibited and the expression of iNOS was elevated 6 weeks after CPZ feeding (Figure 4C, $p<0.001$ respectively), and was effectively induced and declined by GB intervention (Figure 4C, $p<0.01$ respectively), which is consistent with the that of immunohistochemical staining in Figures 4A,B.

In vitro cell experiments, Iba-1 was expressed in cultured BV2 microglia (Figure 5A). The results showed that high concentration of GB $(50 \mu \mathrm{g} / \mathrm{ml})$ inhibited LPS-induced the expression of $\mathrm{p}-\mathrm{NF}-\kappa \mathrm{B} / \mathrm{p} 65$ (Figure $5 \mathrm{~B}, p<0.001$ ), decreased iNOS expression (Figure 5C, $p<0.01$ ) and increased Arg-1 expression (Figure 5C, $p<0.001$ ) compared with control. The results from qPCR showed that relative expression of Arg-1 and iNOS mRNA was up-regulated 6 weeks after CPZ feeding (Figure 5D, $p<0.01$ and $p<0.001$, respectively). GB intervention further increased the relative expression of Arg-1 mRNA and effectively inhibited relative expression of iNOS mRNA (Figure 5D, $p<0.001$ and $p<0.01$, respectively), which is consistent with the that of immunohistochemical staining in Figures 5B,C.

\section{Ginkgolide B Promotes the Production of Neurotrophic Factors From Astrocytes}

Previous studies have shown that neurotrophic factors released by astrocytes promote myelin repair. Compared with mice fed with normal diet, CPZ feeding caused the migration and accumulation of astrocytes in the corpus callosum (Figure 6A, $p<0.001$ ), which can be decreased by GB treatment (Figure 6A, 
A

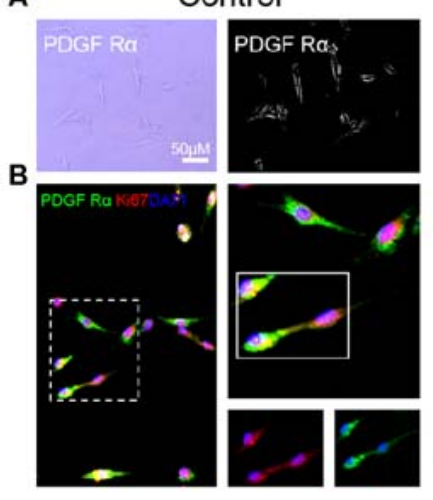

$25 \mu \mathrm{g} / \mathrm{ml} \mathrm{GB}$

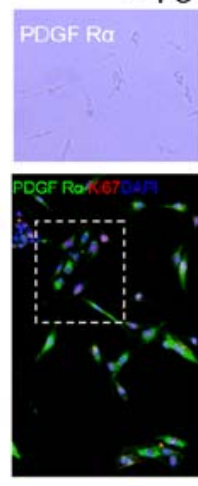

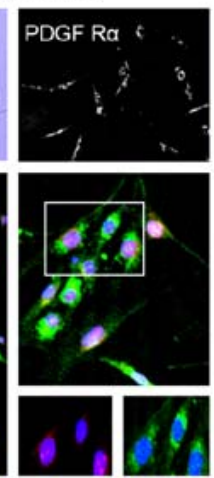

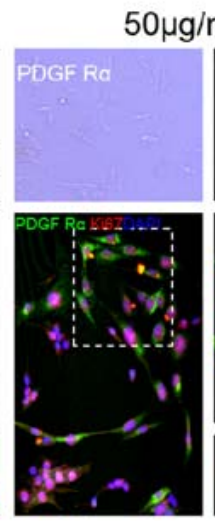

$50 \mu \mathrm{g} / \mathrm{ml} \mathrm{GB}$
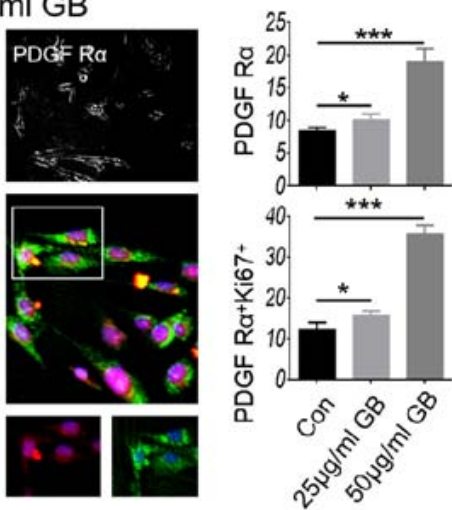

FIGURE 10 | GB (both $25 \mu \mathrm{g} / \mathrm{ml}$ and $50 \mu \mathrm{g} / \mathrm{ml}$ ) promoted the generation of PDGFR $\alpha$ OPCs. (A) Bright field: PDGFR . (B) Double immunocytochemistry staining with anti-PDGFR $\alpha$ and anti-Ki67 in the PDGFR $\alpha$ by immunocytochemistry. Representative images are obtained from three replicate samples/each group, with similar results. The results represent the mean \pm SEM. ${ }^{*} p<0.05,{ }^{* * *} p<0.001$.

$p<0.01)$. We also compared the distribution of astrocytes in other regions of the brain, finding that the numbers of $\mathrm{GFAP}^{+}$ astrocytes in the claustrum, cingulate cortex and cortex of mice fed with CPZ were also elevated as compared with mice fed with normal diet (Figure 6A). The results from Western blot also confirmed that $\mathrm{CPZ}$ feeding induced the expression of GFAP in the brain (Figure 6B, $p<0.001$ ) which can also be inhibited by GB treatment (Figure 6B, $p<0.01$ ). These results suggest that $\mathrm{CPZ}$ feeding induces the migration and activation of astrocytes, while GB treatment effectively inhibits the activation of astrocytes.

To further observe whether GB treatment can induce astrocytes to produce nutrient factors, contributing to the regeneration of myelin sheath, immunohistochemistry was used to detect the expression of BDNF and GDNF on $\mathrm{GFAP}^{+}$ astrocytes. The results showed that GB treatment effectively induced the expression of $\mathrm{BDNF}$ in $\mathrm{GFAP}^{+}$astrocytes in the cingulate cortex and cortex, as compared with mice fed with $\mathrm{CPZ}$ (Figure 7A, $p<0.05$, respectively). Similarly, although $\mathrm{CPZ}$ feeding can slightly induce the expression of GDNF in astrocytes (Figure 7B, $p<0.01$ ), GB treatment further stimulated astrocytes to up-regulate the expression of GDNF in the corpus callosum and subventricular region (Figure $7 \mathbf{B}, p<0.001$ ). The results from ELISA found that CPZ feeding declined the levels of BDNF (1:20 and 1:50) and GDNF (1:20) in the extract of the brain (Figure 7C, $p<0.05$ and $p<0.01$, respectively). GB intervention slightly increased the production of BDNF and GDNF, although the difference was not statistically significant (Figure 7C).

In vitro cell experiments showed that $\mathrm{GB}$ (both $25 \mu \mathrm{g} / \mathrm{ml}$ and $50 \mu \mathrm{g} / \mathrm{ml}$ ) significantly induced the expression of GDNF and $\mathrm{BDNF}$ that were co-localized in $\mathrm{GFAP}^{+}$astrocytes (Figure 8A, $p<0.01, p<0.001$, respectively). At the same time, the results from qPCR showed that relative expression of BDNF and GDNF mRNA was elevated by GB (both $25 \mu \mathrm{g} / \mathrm{ml}$ and $50 \mu \mathrm{g} / \mathrm{ml}$ ) compared with control (Figure 8B, $p<0.001$, respectively).
These results are also similar to those obtained by the in vivo model, indicating that GB might promote astrocytes to secrete neurotrophic BDNF and GDNF in the CPZ model.

\section{Ginkgolide B Promotes the Generation of Oligodendrocyte Precursor Cells}

Oligodendrocyte precursor cells (OPCs), also known as NG2 glia, remain proliferative and continuously generate myelinating oligodendrocytes. In response to a CPZ-induced demyelinating insult, $\mathrm{NG}^{+}$OPCs were rapidly generated in the corpus callosum and lateral septal nucleus of the brain (Figure 9A). However, GB treatment further promoted the generation of $\mathrm{NG} 2^{+}$OPCs in these regions (Figure 9A). In the subventricular zone (SVZ), NG2 ${ }^{+}$OPCs expressing Ki67 were obviously enhanced after GB treatment compared with CPZ-fed mice (Figure 9B, yellow arrow), revealing that GB-induced $\mathrm{NG}^{+}$ OPCs are proliferating.

In vitro cell experiments showed that GB treatment $(25 \mu \mathrm{g}$ and $50 \mu \mathrm{g} / \mathrm{ml}$ ) induced the formation of PDGF Ra+ OPCs (Figures 10A,B, $p<0.05$ and $p<0.001$, respectively). At the same time, low concentration of GB $(25 \mu \mathrm{g} / \mathrm{ml})$ slightly induced the expression of Ki67 by PDGFR $\alpha^{+}$OPCs (Figures 10A,B, $p<0.05)$, while high concentration of GB $(50 \mu \mathrm{g} / \mathrm{ml})$ obviously enhanced PDGFR $\alpha^{+}$OPCs expressing Ki67 (Figures 10A,B, $p<0.001$ ), suggesting that GB induced the formation and proliferation of PDGFR $\alpha^{+}$OPCs.

\section{DISCUSSION}

GB is a terpene lactone found in Ginkgo biloba extract and widely used in the treatment of various neurological diseases, including cerebral ischemia and neurodegeneration. Previous evidence has demonstrated that GB has neuroprotective and anti-inflammatory effects. Here, we observed the therapeutic 


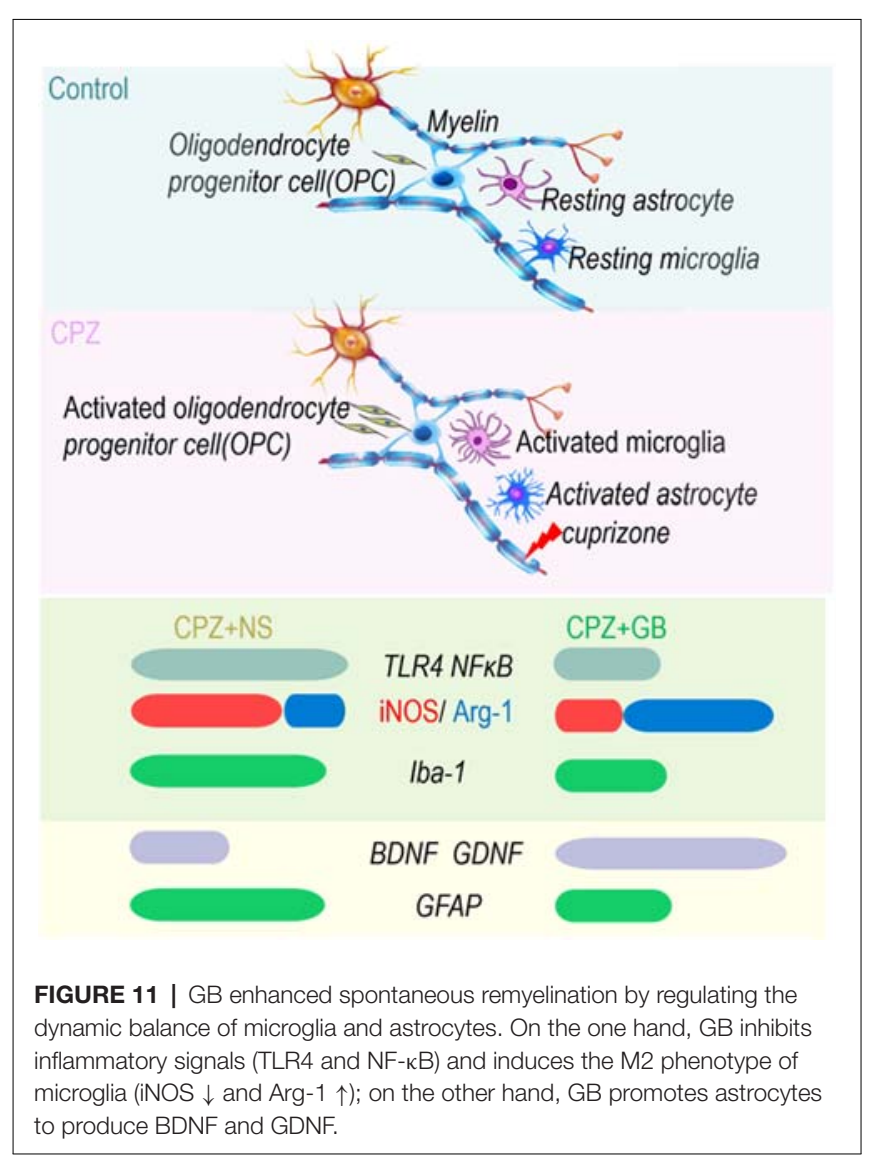

effect of GB in the demyelination model induced by $\mathrm{CPZ}$, manifested by the improvements in behavior and demyelination.

The occurrence and development of MS involve many pathogenic factors, including activation of $\mathrm{T}$ and $\mathrm{B}$ cells, immune triggered inflammation, mitochondrial dysfunction, cell apoptosis and so on. However, glial activation is considered to play a decisive role in the progression of MS (Lassmann, 2014; Mahad et al., 2015). Microglia modulate astrocyte phenotype and function (Rothhammer et al., 2018). The activated microglia release different cytokines (IL-1 $\beta$, IL-6, and TNF- $\alpha$ ) as well as oxidation products ( $\mathrm{NO}$ and ROS), leading to exacerbation of inflammatory cascade and subsequent progression of disease progression (Lassmann et al., 2012; Mahad et al., 2015; Zrzavy et al., 2017). Like the microglia, the A1 subtype of astrocytes is a potent killer of neurons and oligodendrocytes in EAE models and was reported in acute and chronic MS lesions (Liddelow et al., 2017). Nevertheless, the astrocytes in progressive MS play a more specific role in the maintenance of the local inflammation. In fact, the activated microglia can induce neurotoxic reactive astrocytes that contribute to the death of neurons and oligodendrocytes in neurodegenerative disorders, and meanwhile, provide opportunities for the development of new treatments for these diseases (Liddelow et al., 2017).

In a non-immune induced demyelinating model mediated by $\mathrm{CPZ}$, we observed the migration and accumulation of microglia and astrocytes toward the corpus callosum and corresponding myelin sheath regions. A demyelinating event often results not only in the loss of intact myelin sheaths but also the accumulation of myelin debris at the lesion site (Lampron et al., 2015). Inflammatory agents such as lipopolysaccharide (LPS) can stimulate both the migration and phagocytic activity of macrophages (Vallières et al., 2006). It was reported that microglia were activated with increased inflammatory cytokines and myelin phagocytosis (Shobin et al., 2017), indicating that myelin debris may be an inflammatory stimulus for inflammatory microglial activation. During initial demyelination pathology, microglia activation is beneficial since they are involved in myelin debris clearance. However, with the progression of the demyelinating lesion, activated microglia elicit detrimental effects by the overexpression of inflammatory cytokines such as IL- $1 \beta$, IL- 6 , and TNF- $\alpha$ in the surrounding tissue lesion. In this study, we observed that $\mathrm{CPZ}$ feeding induced the inflammatory phenotype of microglia in the Corpus callosum, which should be related to the migration of microglia and phagocytosis of myelin debris. However, it is still unclear whether the decrease of microglia enrichment in the corpus callosum after GB treatment is due to the inhibition of microglia migration and activation by GB or to the acceleration of myelin debris clearance, leading to the remove of microglia from the corpus callosum and the transformation of M2 phenotype.

Previous evidence has demonstrated that astrocytes play dual and controversial roles in demyelinating diseases. The ablation of astrocytes in transgenic mice receiving $\mathrm{CPZ}$ causes a significant decrease in the demyelination within the corpus callosum associated with a reduction in the number of activated microglia in demyelinated sites (Skripuletz et al., 2013). The ablation of astrocytes also reduced the recruitment of microglia as phagocytosing cells to clear the damaged myelin sheath (Skripuletz et al., 2013). These results suggest that astrocytes are harmful to myelin sheath repair. However, astrocytes have the capacity to modify the constituents of the extracellular matrix in MS by producing a variety of nutritional factors that directly influence remyelination within the CNS (Clemente et al., 2013). Consistent with the above results, we found that the treatment of GB promoted the repair of the myelin sheath, accompanied by the up-regulation of BDNF and GDNF expression in astrocytes, suggesting that $\mathrm{GB}$ induces astrocytes to produce neurotrophic factors, which could promote the formation and differentiation of oligodendrocytes in demyelinated regions. BDNF promotes oligodendrocyte differentiation and MBP expression (DuboisDalcq and Murray, 2000; KhorshidAhmad et al., 2016).

At present, we do not elucidate the mechanism by which GB promotes astrocyte to produce neurotrophic factors. It is reported that activated microglia induced neurotoxic reactive astrocytes that resulted in the death of neurons and oligodendrocytes by secreting IL- $1 \alpha$ and TNF- $\alpha$ (Liddelow et al., 2017). IL-1 $\beta$ secreted from microglia is a crucial molecule to alter the functions of astrocytes upon activation of TLR4 (Yan et al., 2019). Therefore, we speculate that GB may maintain the supporting and nutritional functions of astrocytes by inhibiting microglial inflammatory response or inducing microglial M2 polarization in this study.

Oligodendrocyte recruitment and differentiation are regulated by numerous environmental factors, including 
cytokines, chemokines and growth factors (Plemel et al., 2017). In particular, the balance between microglia and astrocytes should create a beneficial microenvironment, which reduces myelin damage and promotes myelin regeneration. Here, we found that $\mathrm{GB}$ can regulate the biological effects of microglia and astrocytes, although it inhibited the enrichment of microglia and astrocytes in the demyelinated areas. GB inhibited the inflammatory phenotype and cytokine production of microglia and induced the growth/nutrient secretion of astrocytes. The former can reduce the further oligodendrocyte damage mediated with the inflammatory microenvironment, while the latter can help the formation and differentiation of oligodendrocytes, achieving a dynamic balance of beneficial microenvironment for myelin repair. Our results also clearly demonstrate that GB treatment promoted the generation of $\mathrm{NG}^{+}$OPCs, which expressed Ki67, suggesting that GB-induced $\mathrm{NG}_{2}^{+}$ OPCs are proliferative and contribute to the remyelination in $\mathrm{CPZ}$-induced demyelination.

In conclusion, $\mathrm{GB}$ treatment protected from $\mathrm{CPZ}$-induced behavior abnormalities, inhibited the inflammatory response, and promoted myelin generation in $\mathrm{CPZ}$ demyelinating model, implicating that GB might act as a novel strategy for the treatment of MS in clinic. The therapeutic potential of GB in CPZ-induced demyelination may be based on the following mechanisms of action: (i) maintains microglia in a deactivated state/M2 phenotype and avoids the deleterious effects of excessive neuroinflammation; and (ii) promotes myelin repair and regeneration by upregulating neurotrophic factors derived from astrocytes (Figure 11). These results highlight the importance of targeting microglia and astrocytes and regulating the dynamic balance of microglia and astrocytes during the remyelination processes. However, the communication and association between microglia and astrocytes mediated with GB still need to be further

\section{REFERENCES}

Acharjee, S., Nayani, N., Tsutsui, M., Hill, M. N., Ousman, S. S., and Pittman, Q. J. (2013). Altered cognitive-emotional behavior in early experimental autoimmune encephalitis - cytokine and hormonal correlates. Brain Behav. Immun. 33, 164-172. doi: 10.1016/j.bbi.2013.07.003

Ahlemeyer, B., and Krieglstein, J. (2003). Neuroprotective effects of Ginkgo biloba extract. Cell. Mol. Life Sci. 60, 1779-1792. doi: 10.1007/s00018-0033080-1

Alamo, A., Condorelli, R. A., La Vignera, S., and Calogero, A. E. (2019). Autoimmune thyroid disease following treatment with alemtuzumab for multiple sclerosis. Int. J. Immunopathol. Pharmacol. 33:2058738419843690. doi: $10.1177 / 2058738419843690$

Chen, A., Xu, Y., and Yuan, J. (2018). Ginkgolide B ameliorates NLRP3 inflammasome activation after hypoxic-ischemic brain injury in the neonatal male rat. Int. J. Dev. Neurosci. 69, 106-111. doi: 10.1016/j. ijdevneu.2018.07.004

Clemente, D., Ortega, M. C., Melero-Jerez, C., and de Castro, F. (2013). The effect of glia-glia interactions on oligodendrocyte precursor cell biology during development and in demyelinating diseases. Front. Cell. Neurosci. 7:268. doi: $10.3389 /$ fncel.2013.00268

Dendrou, C. A., Fugger, L., and Friese, M. A. (2015). Immunopathology of multiple sclerosis. Nat. Rev. Immunol. 15, 545-558. doi: 10.1038/nri3871

Dubois-Dalcq, M., and Murray, K. (2000). Why are growth factors important in oligodendrocyte physiology? Pathol. Biol. 48, 80-86. explored, developing advanced GB therapies to combat neuroinflammation and demyelination in MS and other related pathogenic lesions.

\section{CONCLUSION}

GB promotes myelin generation by regulating the dynamic balance of microglia and astrocytes, but the precise molecular mechanisms are not fully understood, and further studies are required.

\section{DATA AVAILABILITY STATEMENT}

All datasets generated for this study are included in the article.

\section{ETHICS STATEMENT}

The animal study was reviewed and approved by Laboratory and Ethics Committee of Shanxi University of Chinese Medicine.

\section{AUTHOR CONTRIBUTIONS}

J-JY conducted this project and wrote the manuscript. YH and JA carried out Western blot. QM, R-XS and QW participated in data analysis. J-ZY proofread the article. B-GX and C-GM designed the experiments.

\section{FUNDING}

This work was supported by grants from the National Natural Science Foundation of China (Nos. 81473577 and 81371414), and Research Project Supported by Shanxi Scholarship Council of China (2014-7).

Friese, M. A., Schattling, B., and Fugger, L. (2014). Mechanisms of neurodegeneration and axonal dysfunction in multiple sclerosis. Nat. Rev. Neurol. 10, 225-238. doi: 10.1038/nrneurol.2014.37

Granberg, T., Fan, Q., Treaba, C. A., Ouellette, R., Herranz, E., Mangeat, G., et al. (2017). In vivo characterization of cortical and white matter neuroaxonalpathology in early multiple sclerosis. Brain 140, 2912-2926. doi: 10.1093/brain/awx247

Ibitoye, R., Kemp, K., Rice, C., Hares, K., Scolding, N., and Wilkins, A. (2016). Oxidative stress-related biomarkers in multiple sclerosis: a review. Biomark. Med. 10, 889-902. doi: 10.2217/bmm-2016-0097

KhorshidAhmad, T., Acosta, C., Cortes, C., Lakowski, T. M., Gangadaran, S., and Namaka, M. (2016). Transcriptional regulation of brain-derived neurotrophic factor (BDNF) by Methyl CpG binding protein 2 (MeCP2): a novel mechanism for re-myelination and/or myelin repair involved in the treatment of multiple sclerosis (MS). Mol. Neurobiol. 53, 1092-1107. doi: 10.1007/s12035-014-9074-1

Klotz, L., Havla, J., Schwab, N., Hohlfeld, R., Barnett, M., Reddel, S., et al. (2019). Risks and risk management in modern multiple sclerosis immunotherapeutic treatment. Ther. Adv. Neurol. Disord. 12:1756286419836571. doi: 10.1177/1756286419836571

Lampron, A., Larochelle, A., Laflamme, N., Préfontaine, P., Plante, M. M., Sánchez, M. G., et al. (2015). Inefficient clearance of myelin debris by microglia impairs remyelinating processes. J. Exp. Med. 212, 481-495. doi: 10.1084/jem. 20141656

Lassmann, H. (2014). Mechanisms of white matter damage in multiple sclerosis. Glia 62, 1816-1830. doi: 10.1002/glia.22597 
Lassmann, H., van Horssen, J., and Mahad, D. (2012). Progressive multiple sclerosis: pathology and pathogenesis. Nat. Rev. Neurol. 8, 647-656. doi: 10.1038/nrneurol.2012.168

Li, W., Qinghai, S., Kai, L., Xue, M., Lili, N., Jihua, R., et al. (2019). Oral administration of Ginkgolide B alleviates hypoxia-induced neuronal damage in rat hippocampus by inhibiting oxidative stress and apoptosis. Iran. J. Basic Med. Sci. 22, 140-145. doi: 10.22038/ijbms.2018.26228.6569

Liddelow, S. A., Guttenplan, K. A., Clarke, L. E., Bennett, F. C., Bohlen, C. J., Schirmer, L., et al. (2017). Neurotoxic reactive astrocytes are induced by activated microglia. Nature 541, 481-487. doi: 10.1038/nature21029

Mahad, D. H., Trapp, B. D., and Lassmann, H. (2015). Pathological mechanisms in progressive multiple sclerosis. Lancet Neurol. 14, 183-193. doi: 10.1016/s14744422(14)70256-x

Plemel, J. R., Liu, W. Q., and Yong, V. W. (2017). Remyelination therapies: a new direction and challenge in multiple sclerosis. Nat. Rev. Drug Discov. 16, 617-634. doi: 10.1038/nrd.2017.115

Qin, Y., Zhang, Y., Tomic, I., Hao, W., Menger, M. D., Liu, C., et al. (2018). Ginkgo biloba extract EGb 761 and its specific components elicit protective protein clearance through the autophagy-lysosomal pathway in Tau-transgenic mice and cultured neurons. J. Alzheimers Dis. 65, 243-263. doi: 10.3233/jad-180426

Rajda, C., Pukoli, D., Bende, Z., Majláth, Z., and Vécsei, L. (2017). Excitotoxins, mitochondrial and redox disturbances in multiple sclerosis. Int. J. Mol. Sci. 18:E353. doi: 10.3390/ijms18020353

Reich, D. S., Lucchinetti, C. F., and Calabresi, P. A. (2018). Multiple sclerosis. N. Engl. J. Med. 378, 169-180. doi: 10.1056/NEJMra1401483

Rothhammer, V., Borucki, D. M., Tjon, E. C., Takenaka, M. C., Chao, C. C., Ardura-Fabregat, A., et al. (2018). Microglial control of astrocytes in response to microbial metabolites. Nature 557, 724-728. doi: 10.1038/s41586-0180119-x

Shobin, E., Bowley, M. P., Estrada, L. I., Heyworth, N. C., Orczykowski, M. E., Eldridge, S. A., et al. (2017). Microglia activation and phagocytosis: relationship with aging and cognitive impairment in the rhesus monkey. Geroscience 39, 199-220. doi: 10.1007/s11357-017-9965-y

Skripuletz, T., Hackstette, D., Bauer, K., Gudi, V., Pul, R., Voss, E., et al. (2013). Astrocytes regulate myelin clearance through recruitment of microglia during cuprizone-induced demyelination. Brain 136, 147-167. doi: 10.1093/brain/aws262

Stone, S., and Lin, W. (2015). The unfolded protein response in multiple sclerosis. Front. Neurosci. 9:264. doi: 10.3389/fnins.2015.00264

Thompson, A. J., Baranzini, S. E., Geurts, J., Hemmer, B., and Ciccarelli, O. (2018). Multiple sclerosis. Lancet 391, 1622-1636. doi: 10.1016/S0140-6736(18) 30481-1

Vallières, N., Berard, J. L., David, S., and Lacroix, S. (2006). Systemic injections of lipopolysaccharide accelerates myelin phagocytosis during Wallerian degeneration in the injured mouse spinal cord. Glia 53, 103-113. doi: 10.1002/glia.20266

Vaughn, C., Bushra, A., Kolb, C., and Weinstock-Guttman, B. (2018). An update on the use of disease-modifying therapy in pregnant patients with multiple sclerosis. CNS Drugs 32, 161-178. doi: 10.1007/s40263-0180496-6

Vega-Riquer, J. M., Mendez-Victoriano, G., Morales-Luckie, R. A., and Gonzalez-Perez, O. (2019). Five decades of cuprizone, an updated model to replicate demyelinating diseases. Curr. Neuropharmacol. 17, 129-141. doi: 10.2174/1570159x15666170717120343

Velter, C., Thomas, M., Cavalcanti, A., Bastien, M., Chochon, F., Lubetzki, C., et al. (2019). Melanoma during fingolimod treatment for multiple sclerosis. Eur. J. Cancer 113, 75-77. doi: 10.1016/j.ejca.2019. 03.011

Xu, X., Dai, J., Liu, C., Chen, Y., Xin, F., Zhou, F., et al. (2019). Common and disorder-specific neurofunctional markers of dysregulated empathic reactivity in major depression and generalized anxiety disorder. Psychother. Psychosom. 26, 1-3. doi: 10.1159/000504180

Yan, X., Li, F., Maixner, D. W., Yadav, R., Gao, M., Ali, M. W., et al. (2019). Interleukin-1 $\beta$ released by microglia initiates the enhanced glutamatergic activity in the spinal dorsal horn during paclitaxel-associated acute pain syndrome. Glia 67, 482-497. doi: 10.1002/glia.23557

Zhen, W., Liu, A., Lu, J., Zhang, W., Tattersall, D., and Wang, J. (2017). An alternative cuprizone-induced demyelination and remyelination mouse model. ASN Neuro 9:1759091417725174. doi: 10.1177/17590914177 25174

Zheng, P. D., Mungur, R., Zhou, H. J., Hassan, M., Jiang, S. N., and Zheng, J. S. (2018). Ginkgolide B promotes the proliferation and differentiation of neural stem cells following cerebral ischemia/reperfusion injury, both in vivo and in vitro. Neural Regen. Res. 13, 1204-1211. doi: 10.4103/1673-5374. 232476

Zimmermann, T., Bartsch, J. C., Beer, A., Lomazzo, E., Guggenhuber, S., Lange, M. D., et al. (2019). Impaired anandamide/palmitoylethanolamide signaling in hippocampal glutamatergic neurons alters synaptic plasticity, learning, and emotional responses. Neuropsychopharmacology 44, 1377-1388. doi: 10.1038/s41386-018-0274-7

Zrzavy, T., Hametner, S., Wimmer, I., Butovsky, O., Weiner, H. L., and Lassmann, H. (2017). Loss of 'homeostatic' microglia and patterns of their activation in active multiple sclerosis. Brain 140, 1900-1913. doi: 10.1093/brain/awx113

Conflict of Interest: The authors declare that the research was conducted in the absence of any commercial or financial relationships that could be construed as a potential conflict of interest.

Copyright (C) 2020 Yin, He, An, Miao, Sui, Wang, Yu, Xiao and Ma. This is an open-access article distributed under the terms of the Creative Commons Attribution License (CC BY). The use, distribution or reproduction in other forums is permitted, provided the original author(s) and the copyright owner(s) are credited and that the original publication in this journal is cited, in accordance with accepted academic practice. No use, distribution or reproduction is permitted which does not comply with these terms. 